\title{
A kinematical approach to dark energy studies
}

\author{
David Rapetti ${ }^{1 \star}$, Steven W. Allen ${ }^{1}$, Mustafa A. Amin ${ }^{1}$, Roger D. Blandford ${ }^{1}$ \\ ${ }^{1}$ Kavli Institute for Particle Astrophysics and Cosmology, Stanford University, 382 Via Pueblo Mall, Stanford 94305-4060, USA.
}

Accepted ???, Received ???; in original form 29 May 2006

\begin{abstract}
We present and employ a new kinematical approach to cosmological 'dark energy' studies. We construct models in terms of the dimensionless second and third derivatives of the scale factor $a(t)$ with respect to cosmic time $t$, namely the present-day value of the deceleration parameter $q_{0}$ and the cosmic jerk parameter, $j(t)$. An elegant feature of this parameterization is that all $\Lambda \mathrm{CDM}$ models have $j(t)=1$ (constant), which facilitates simple tests for departures from the $\Lambda$ CDM paradigm. Applying our model to the three best available sets of redshift-independent distance measurements, from type Ia supernovae and X-ray cluster gas mass fraction measurements, we obtain clear statistical evidence for a late time transition from a decelerating to an accelerating phase. For a flat model with constant jerk, $j(t)=j$, we measure $q_{0}=-0.81 \pm$ 0.14 and $j=2.16_{-0.75}^{+0.81}$, results that are consistent with $\Lambda \mathrm{CDM}$ at about the $1 \sigma$ confidence level. A standard 'dynamical' analysis of the same data, employing the Friedmann equations and modeling the dark energy as a fluid with an equation of state parameter, $w$ (constant), gives $\Omega_{\mathrm{m}}=0.306_{-0.040}^{+0.042}$ and $w=-1.15_{-0.18}^{+0.14}$, also consistent with $\Lambda \mathrm{CDM}$ at about the $1 \sigma$ level. In comparison to dynamical analyses, the kinematical approach uses a different model set and employs a minimum of prior information, being independent of any particular gravity theory. The results obtained with this new approach therefore provide important additional information and we argue that both kinematical and dynamical techniques should be employed in future dark energy studies, where possible. Our results provide further interesting support for the concordance $\Lambda \mathrm{CDM}$ paradigm.
\end{abstract}

Key words: cosmology:observations - cosmology:cosmological parameters - cosmology:theory - supernovae - x-ray clusters - dark energy

\section{INTRODUCTION}

The field of cosmology has made unprecedented progress during the past decade. This has largely been driven by new observations, including precise measurements of the spectrum of cosmic microwave background (CMB) anisotropies (Spergel et al. 2003; Spergel et al. 2006, and references therein), the distance-redshift relation to type Ia supernovae (Riess et al. 1998; Perlmutter et al. 1999; Knop et al. 2003; Riess et al. 2004; Astier et al. 2005), the distance-redshift relation to X-ray galaxy clusters (Allen et al. 2002, 2004; Ettori et al. 2003), measurements of the mean matter density and amplitude of matter fluctuations from X-ray clusters (Borgani et al. 2001; Allen et al. 2003; Reiprich \& Böhringer 2002; Schuecker et al. 2003; Voevodkin \& Vikhlinin 2004), measurements of the matter power spectrum from galaxy redshift surveys (Tegmark et al. 2004; Cole et al. 2005; Eisenstein et al. 2005), Lyman- $\alpha$ forest studies (Croft et al. 2002;

* Email: drapetti@slac.stanford.edu
Viel et al. 2004; Seljak et al. 2005) and weak lensing surveys (van Waerbeke et al. 2000; Hoekstra et al. 2002; Van Waerbeke et al. 2005; Jarvis et al. 2005), and measurements of the Integrated Sachs-Wolfe effect (Scranton et al. 2003; Fosalba et al. 2003).

These and other experiments have lead to the definition of the so-called concordance $\Lambda \mathrm{CDM}$ cosmology. In this model, the Universe is geometrically flat with only $\sim 4$ per cent of the current mass-energy budget consisting of normal baryonic matter. Approximately 23 per cent is cold dark matter, which interacts only weakly with normal baryonic matter but which clusters under the action of gravity. The remaining $\sim 73$ per cent consists of smoothly distributed quantum vacuum energy (the cosmological constant), which pushes the Universe apart. This combination of matter and vacuum energy leads to the expectation that the Universe should undergo a late time transition from a decelerating to an accelerating phase of expansion. Late-time acceleration of the Universe is now an observed fact (e.g. Riess et al. 2004; Allen et al. 2004; Astier et al. 2005). A transition from a decelerating phase to a late-time accelerating phase is required 
to explain both these late-time acceleration measurements and the observed growth of structure.

Despite the observational success of the concordance $\Lambda \mathrm{CDM}$ model, significant fine tuning problems exist. In particular, difficulties arise in adjusting the density of the vacuum energy to be a non-zero but tiny number, when compared with the value predicted by standard theoretical calculations, and with explaining why the current matter and vacuum energy densities are so similar (the 'cosmic coincidence' problem). For these reasons, amongst others, a large number of alternative cosmological models have been proposed. These include models that introduce new energy components to the Universe - so called 'dark energy' models e.g. scalar 'quintessence' fields (Caldwell et al. 1998; Zlatev et al. 1999; Copeland et al. 1998; Steinhardt et al. 1999; Barreiro et al. 2000), K-essence (Chiba et al. 2000; Armendariz-Picon et al. 2000, 2001), tachyon fields (Bagla et al. 2003; Copeland et al. 2005) and Chaplygin gas models (Kamenshchik et al. 2001; Bento et al. 2002). Other possibilities include modified gravity theories, motivated by e.g. the existence of extra dimensions (Dvali et al. 2000; Deffayet et al. 2002; Deffayet et al. 2002; Maartens \& Majerotto 2006; Guo et al. 2006) or other modifications of General Relativity (Capozziello et al. 2003; Carroll et al. 2004; Vollick 2003; Carroll et al. 2005; Mena et al. 2006; Navarro \& Van Acoleyen 2005), which can also lead to late-time cosmic acceleration. The simplicity of the concordance $\Lambda \mathrm{CDM}$ model, however, makes it highly attractive. A central goal of modern observational cosmology is to test whether this model continues to provide an adequate description of rapidly improving data.

Most current analyses of cosmological data assume General Relativity and employ the mean matter density of the Universe, $\Omega_{\mathrm{m}}$, and the dark energy equation of state $w$ as model parameters. Such analyses are often referred to as 'dynamical studies', employing as they do the Friedmann equations. Other dynamical analyses employ modified Friedmann equations for a particular gravity model. However, a purely kinematical approach is also possible that does not assume any particular gravity theory. Kinematical models provide important, complementary information when seeking to understand the origin of the observed late-time accelerated expansion.

In a pioneering study, Riess et al. (2004) measured a transition from a decelerating to accelerating phase using a simple linear parameterization of the deceleration parameter $q(z)$, where $q(z)$ is the dimensionless second derivative of the scale factor, $a(t)$, with respect to cosmic time. Recently, Shapiro \& Turner (2005), Gong \& Wang (2006) and Elgaroy \& Multamaki (2006) have employed a variety of other parameterizations, constructed in terms of $q(z)$, to study this transition. However, since the underlying physics of the transition are unknown, the choice of a particular parameterization for $q(z)$ is quite arbitrary. Shapiro \& Turner (2005) applied a principal component analysis of $q(z)$ to the supernovae data of Riess et al. (2004) and found strong evidence for recent, changing acceleration but weak evidence for a decelerated phase in the past (i.e. weak evidence for a transition between the two phases). Elgaroy \& Multamaki (2006) employed a Bayesian analysis to the Riess et al. (2004) data and the more recent SNLS supernovae sample of Astier et al. (2005), obtaining a similar result.

In this paper we develop an improved method for study- ing the kinematical history of the Universe. Instead of using parameterizations constructed in terms of $q(z)$, we follow Blandford et al. (2004) and introduce the cosmic jerk, $j(a)$, the dimensionless third derivative of the scale factor with respect to cosmic time. (Here $a$ is the cosmic scale factor, with $a=1 / 1+z$.) The use of the cosmic jerk formalism provides a more natural parameter space for kinematical studies. Our results are presented in terms of current deceleration parameter $q_{0}$ and $j(a)$, where the latter can be either constant or evolving. We apply our method to the three best current kinematical data sets: the'gold' sample of type Ia supernovae (hereafter SNIa) measurements of Riess et al. (2004), the SNIa data from the first year of the Supernova Legacy Survey (SNLS) project (Astier et al. 2005), and the X-ray galaxy cluster distance measurements of Allen et al. (2006). This latter data set is derived from measurements of the baryonic mass fraction in the largest relaxed galaxy clusters, which is assumed to be a standard quantity for such systems (see e.g. Allen et al. 2004, for discussion).

In General Relativity $j(a)$ depends in a non-trivial way on both $\Omega_{\mathrm{m}}$ and $w(a)$ (Blandford et al. 2004). In general, there is no one-to-one mapping between models with constant $j$ and models with constant $w$. A powerful feature of the standard dynamical approach is that all $\Lambda$ CDM models have $w=-1$ which make it easy to search for departures from $\Lambda$ CDM. Likewise, the use of the jerk formalism imbues the kinematical analysis with a similar important feature in that all $\Lambda \mathrm{CDM}$ models are represented by a single value of $j=1$. The use of the jerk formalism thus enables us to constrain and explore departures from $\Lambda \mathrm{CDM}$ in the kinematical framework in an equally effective manner. Moreover, by employing both the dynamical and kinematical approaches to the analysis of a single data set, we explore a wider set of questions than with a single approach. We note that Sahni et al. (2003) and Alam et al. (2003) also drew attention to the importance of the jerk parameter for discriminating models of dark energy and/or modified gravity.

Using the three kinematical data sets mentioned above, we find clear evidence for a negative value of $q_{0}$ (current acceleration) and a positive cosmic jerk, assuming $j$ constant. The concordance $\Lambda \mathrm{CDM}$ model provides a reasonable description of the data, using both the new kinematical and standard dynamical approaches. We also search for more complicated deviations from $\Lambda \mathrm{CDM}$, allowing $j(a)$ to evolve as the Universe expands, in an analogous manner to dynamical studies which allow from time-variation of the dark energy equation of state $w(a)$. Our analysis employs a Chebyshev polynomial expansion and a Markov Chain Monte Carlo approach to explore parameter spaces. We find no evidence for a time-varying jerk.

This paper is structured as follows: in section 2 we describe our new kinematical approach. In section 3 we describe the scheme adopted for polynomial expansions of $j(a)$. Section 4 includes details of the data analysis. The results from the application of our method to the supernovae and $\mathrm{X}$-ray cluster data are presented in section 5. Finally, our main conclusions are summarized in section 7 . Throughout this paper, we assume that the Universe is geometrically flat. 


\section{THE KINEMATICAL AND DYNAMICAL FRAMEWORKS FOR LATE TIME COSMIC ACCELERATION}

\subsection{Previous work}

The expansion rate of the Universe can be written in terms of the Hubble parameter, $H=\dot{a} / a$, where $a$ is the scale factor and $\dot{a}$ is its first derivative with respect to time. The current value of the Hubble parameter is the Hubble Constant, usually written as $H_{0}$. Under the action of gravity, and for negligible vacuum energy, the expansion of the Universe is expected to decelerate at late times. Contrary to this expectation, in the late 1990s, type Ia supernovae experiments (Riess et al. 1998; Perlmutter et al. 1999) provided the first direct evidence for a late time accelerated expansion of the Universe. In particular, the present value of the deceleration parameter, $q_{0}$, measured from the supernova data was found to be negative. In detail, the deceleration parameter $q$ is defined as the dimensionless second derivative of the scale factor

$q(t)=-\frac{1}{H^{2}}\left(\frac{\ddot{a}}{a}\right)$,

and in terms of the scale factor,

$q(a)=-\frac{1}{H}(a H)^{\prime}$

where the 'dots' and 'primes' denote derivatives with respect to cosmic time and scale factor, respectively.

The current 'concordance' cosmological model, $\Lambda \mathrm{CDM}$, has been successful in explaining the SNIa results and all other precision cosmology measurements to date. Together with it's theoretical simplicity, this makes the $\Lambda$ CDM model very attractive. However, as discussed in the introduction, the concordance model does face significant theoretical challenges and a wide-range of other possible models also provide adequate descriptions of the current data (see Copeland et al. 2006, for an extensive review).

An excellent way to distinguish between models is to obtain precise measurements of the time evolution of the expansion of the Universe. Given such data, a number of different analysis approaches are possible. In searching for time evolution in the deceleration parameter, as measured by current SNIa data, Riess et al. (2004) assumed a linear parameterization of $q(z)$,

$q(z)=q_{0}+\frac{d q}{d z} z$

These authors measured a change in sign of the deceleration parameter, from postive to negative approaching the present day, at a redshift $z_{\mathrm{t}}=0.46 \pm 0.13$. Using this parameterization for $q(z)$, the definition of the deceleration parameter given by equation (1), and integrating over the redshift, we obtain that for this model the evolution of the Hubble parameter

$E(z)=H(z) / H_{0}=(1+z)^{\left(1+q_{0}-q^{\prime}\right)} e^{q^{\prime} z}$,

where $q^{\prime}=d q / d z$.

However, since the origin of cosmic acceleration is unknown, it is important to recognize that the choice of any particular parameterized expansion for $q(z)$ is essentially arbitrary. Indeed, when (or if) a transition between decelerated and accelerated phases if inferred to occur can depend on the parameterization used. Elgaroy \& Multamaki (2006) showed that using the linear parameterization described by equation (3) and fitting to the SNIa data set of Astier et al. (2005) a transition redshift $z_{\mathrm{t}} \sim 2.0$ is obtained which, uncomfortably, lies beyond the range of the data used.

Transitions between phases of different cosmic acceleration are more naturally described by models incorporating a cosmic 'jerk'. The jerk parameter, $j(a)$, is defined as the dimensionless third derivative of the scale factor with respect to cosmic time (Blandford et al. 2004)

$j(t)=\frac{1}{H^{3}}\left(\frac{\dot{a}}{a}\right)$

and in terms of the scale factor

$j(a)=\frac{\left(a^{2} H^{2}\right)^{\prime \prime}}{2 H^{2}}$

where again the 'dots' and 'primes' denote derivatives with respect to cosmic time and scale factor, respectively.

In such models, a transition from a decelerating phase at early times to an accelerating phase at late times occurs for all models with $q_{0}<0$ and a positive cosmic jerk. Note that a Taylor expansion of the Hubble parameter around small redshifts (Visser 2004; Riess et al. 2004) contains the present value of both the deceleration and jerk parameters, $q_{0}$ and $j_{0}$. Such Taylor expansions are inappropriate for fitting highredshift objects (Blandford et al. 2004; Linder 2006), such as those included in the data sets used here.

Blandford et al. (2004) describe how the jerk parameterization provides a convenient, alternative method to describe models close to $\Lambda \mathrm{CDM}$. In this parameterization, flat $\Lambda \mathrm{CDM}$ models have a constant jerk with $j(a)=1$ (note that this neglects the effects of radiation over the redshift range of interest, which is also usually the case when modeling within the dynamic framework). Thus, any deviation from $j=1$ measures a departure from $\Lambda$ CDM, just as deviations from $w=-1$ do in more standard dynamical analyses. Importantly, in comparison to dynamical approaches, however, the kinematical approach presented here explores both a different set of models and imposes fewer assumptions. The dynamical approach has other strengths, however, and can be applied to a wider range of data (e.g. CMB and growth of structure studies), making the kinematical and dynamical approaches highly complementary.

It is interesting to note that, in principle, any particular dynamical parameter space will have its own physical limits. For instance, within the dynamical $\left(\Omega_{\mathrm{m}}, w\right)$ plane, models with $w<-1$, known as 'phantom' dark energy models, violate the dominant energy condition (Carroll et al. 2003) and present serious problems relating to the treatment of dark energy perturbations (Hu 2005; Vikman 2005; Caldwell \& Doran 2005; Zhao et al. 2005) when $w(z)$ crosses the boundary $w=-1$. Current data allow models with $w<-1$ (Weller \& Lewis 2003; Riess et al. 2004; Allen et al. 2004; Astier et al. 2005; Spergel et al. 2006; Cabre et al. 2006) and models in which $w(z)$ crosses the boundary $w=-1$ (Jassal et al. 2005; Corasaniti et al. 2004; Seljak et al. 2005; Rapetti et al. 2005; Upadhye et al. 2005; Zhao et al. 2006). However, another dynamical parameter space, coming e.g. from a different gravity theory, might not pathologically suffer from such boundaries around the models allowed for current data. 
Since the $\left(q_{0}, j\right)$ plane (see below) is purely kinematical, i.e. no particular gravity theory is assumed, we are not forced to interpret $j=1$, or any locus in this plane, as a barrier. Note, however, that caution is required in extending the results from the kinematical analysis beyond the range of the observed data (see Amin et al. 2006, for details). For example, inappropriately extending a jerk model to very high redshifts could imply an unphysical Hubble parameter at early times.

\subsection{A new kinematical framework}

For our kinematical analysis, we first calculate $H(a)$ given $j(a ; \mathcal{C})$ where $\mathcal{C}=\left(c_{0}, c_{1}, \ldots, c_{\mathrm{N}}\right)$ is the selected vector of parameters used to describe the evolution of $j(a)$ (see below). Following Blandford et al. (2004) we rewrite the defining equation for the jerk parameter (6) in a more convenient form

$a^{2} V^{\prime \prime}(a)-2 j(a) V(a)=0$

where ' denotes derivative with respect to $a$ and $V(a)$ is defined as

$V(a)=-\frac{a^{2} H^{2}}{2 H_{0}^{2}}$.

We specify the two constants of integration required by (7) in terms of the present Hubble parameter $H_{0}$ and the present deceleration parameter $q_{0}$ as follows

$V(1)=-\frac{1}{2}, \quad V^{\prime}(1)=q_{0}$,

where $a\left(t_{0}\right)=1$ at the present time $t_{0}$. Here the first condition comes from $H(1)=H_{0}$ and the second from

$V^{\prime}(1)=-\frac{H_{0}^{\prime}}{H_{0}}-1=q_{0}$

The Hubble parameter, $H(a)$, obtained from equations $(7),(8)$ and (9) is used to calculate the angular diameter $\left(d_{\mathrm{A}}\right)$ and luminosity $\left(d_{\mathrm{L}}\right)$ distances for a flat FriedmannRobertson-Walker-Lemaître (FRWL) metric

$d_{\mathrm{A}}(a)=a^{2} d_{\mathrm{L}}(a)=\frac{c}{H_{0}} a \int_{a}^{1} \frac{1}{a^{2} E(a)} d a$,

where $c$ is the speed of light. These theoretical distances $d_{\mathrm{L}}(a)$ and $d_{\mathrm{A}}(a)$ are then used in the data analysis (see section 4).

Our framework provides a simple and intuitive approach for kinematical studies. For models with $q_{0}<0(>0)$, the Universe is currently accelerating (decelerating). Models with $q_{0}<0$ and $j(a)=1$ (constant) are currently accelerating and have the expansion evolving in a manner consistent with $\Lambda$ CDM. Any significant departure from $j=1$ indicates that some other mechanism is responsible for the acceleration.

\subsection{Standard dynamical framework}

For comparison purposes, we have also carried out a standard dynamical analysis of the data in which we employ a dark energy model with a constant dark energy equation of state, $w$. From energy conservation of the dark energy fluid and the Friedmann equation, we obtain the evolution of the Hubble parameter, $H(z)=H_{0} E(z)$,
$E(z)=\left[\Omega_{\mathrm{m}}(1+z)^{3}+\left(1-\Omega_{\mathrm{m}}\right)(1+z)^{3(1+w)}\right]^{1 / 2}$,

where $\Omega_{\mathrm{m}}$ is the mean matter density in units of the critical density. As with the kinematical analysis, we assume flatness and neglect the effects of radiation density. In this framework, models with a cosmological constant have $w=-1$ at all times.

\section{EVOLVING JERK MODELS}

Our analysis allows for the possibility the cosmic jerk parameter, $j(a)$ may evolve with the scale factor. We have restricted our analysis to the range of $a$ where we have data, $\left[a_{\min }=0.36, a_{\max }=1\right]$. In searching for possible evolution, our approach is to adopt $\Lambda$ CDM as a base model and search for progressively more complicated deviations from this. We begin by allowing a constant deviation $\Delta j$ from $\Lambda \mathrm{CDM}(j=1)$. For this model, it is possible to solve the jerk equation (7) analytically. Using the initial conditions listed in (9), we obtain

$V(a)=-\frac{\sqrt{a}}{2}\left[\left(\frac{p-u}{2 p}\right) a^{p}+\left(\frac{p+u}{2 p}\right) a^{-p}\right]$

where $p \equiv(1 / 2) \sqrt{(1+8 j)}$ and $u \equiv 2\left(q_{0}+1 / 4\right)$.

For the next most complicated possible deviation from $\Lambda \mathrm{CDM}$, we have $j(a ; \mathcal{C})=j^{\Lambda \mathrm{CDM}}+\Delta j(a ; \mathcal{C})$. Here $j^{\Lambda \mathrm{CDM}}=$ 1 and $j(a ; \mathcal{C})$ is the cosmic jerk for the cosmology in question. In order to meaningfully increase the number of parameters in the vector $\mathcal{C}$, we employ a framework constructed from Chebyshev polynomials. The Chebyshev polynomials form a basis set of polynomials that can be used to approximate a given function over the interval $[-1,1]$. We rescale this interval to locate our function $\Delta j(a ; \mathcal{C})$ in the range of scale factor where we have data:

$a_{c} \equiv \frac{a-\frac{1}{2}\left(a_{\min }+a_{\max }\right)}{\frac{1}{2}\left(a_{\max }-a_{\min }\right)}$,

where $a$ is the scale factor in the range of interest and $a_{\mathrm{c}}$ is Chebyshev variable. The trigonometric expression for a Chebyshev polynomial of degree $n$ is given by

$T_{\mathrm{n}}\left(a_{\mathrm{c}}\right)=\cos \left(n \arccos a_{\mathrm{c}}\right)$.

These polynomials can also be calculated using the recurrent formula

$T_{\mathrm{n}+1}\left(a_{\mathrm{c}}\right)=2 a_{\mathrm{c}} T_{\mathrm{n}}\left(a_{\mathrm{c}}\right)-T_{\mathrm{n}-1}\left(a_{\mathrm{c}}\right), \quad n \geqslant 1$,

where $T_{0}\left(a_{\mathrm{c}}\right)=1$ and, for example, the next three orders are $T_{1}\left(a_{\mathrm{c}}\right)=a_{\mathrm{c}}, T_{2}\left(a_{\mathrm{c}}\right)=2{a_{\mathrm{c}}}^{2}-1, T_{3}\left(a_{\mathrm{c}}\right)=4 a_{\mathrm{c}}{ }^{3}-3 a_{\mathrm{c}}$, etc. Using a weighted combination of these components, any arbitrary function can be approximately reconstructed. The underlying deviation from $\Lambda \mathrm{CDM}$ can be expressed as

$\Delta j(a ; \mathcal{C}) \simeq \sum_{\mathrm{n}=0}^{\mathrm{N}} c_{\mathrm{n}} T_{\mathrm{n}}\left(a_{\mathrm{c}}\right)$

where the weighting coefficients form our vector of parameters, $\mathcal{C}=\left(c_{0}, c_{1}, \ldots, c_{\mathrm{N}}\right)$. Thus, using equation (17) we produce different parameterizations for increasing $N$. With higher $N$ 's we allow a more precise exploration of the $\left[q_{0}, j(a ; \mathcal{C})\right]$ parameter space. However, it is clear that this process will be limited by the ability of the current data to distinguish between such models. In order to judge how 
many orders of polynomials to include, we quantify the improvements to the fits obtained from the inclusion of progressively higher orders in a variety of ways (see below). In general, we find that models with a degree of complexity beyond a constant jerk are not required by current data.

We note that approches other than expanding $\Delta j$ in Chebyshev polynomials are possible, e.g. one could include the dimensionless fourth derivative of the scale factor as a model parameter. However, since $\Lambda$ CDM does not make any special prediction for the value of this derivative, we prefer to use our general expansion in $\Delta j$ here.

\section{DATA AND ANALYSIS METHODS}

\subsection{Type Ia supernovae data}

For the analysis of SNIa data, we use both the 'gold' sample of Riess et al. (2004) and the first year SNLS sample of Astier et al. (2005). The former data set contains $157^{1}$ SNIa, where a subset of 37 low-redshift objects are in common with the data of Astier et al. (2005). Astier et al. (2005) contains $115^{2}$ objects. We use the measurements of Astier et al. (2005) for objects in common between the studies. Thus, combining both data sets we have 120 SNIa from the Riess et al. (2004) gold sample (157 minus the 37 low-redshift objects in common) and 115 SNIa from Astier et al. (2005).

The two SNIa studies use different light-curve fitting methods. In order to compare and combine the data, we fit the observed distance moduli $\mu^{\text {obs }}\left(z_{\mathrm{i}}\right)=m^{\text {obs }}\left(z_{\mathrm{i}}\right)-M$, where $m$ is the apparent magnitude at maximum light after applying galactic extinction, K-correction and light curve widthluminosity corrections, and $M$ is the absolute magnitude, with the theoretical predictions, $\mu^{\text {th }}\left(z_{\mathrm{i}}\right)=m^{\text {th }}\left(z_{\mathrm{i}}\right)-M=$ $5 \log _{10} D_{\mathrm{L}}\left(z_{\mathrm{i}} ; \theta\right)+\mu_{0}$, where $D_{\mathrm{L}}=H_{0} d_{\mathrm{L}}$ is the $H_{0}$-free luminosity distance, $\mu_{0}=25-5 \log _{10} H_{0}$ and $m_{0} \equiv M+\mu_{0}$ is a "nuisance parameter" which contains both the absolute magnitude and $H_{0}$.

For the $\left[q_{0}, j(a ; \mathcal{C})\right]$ parameter space, the luminosity distance $d_{\mathrm{L}}(z ; \theta)$ is directly obtained integrating the solution of the differential equation (7) with the definition (11) as presented in subsection 2.2. For models using linear parameterization of $q(z)$ and/or dynamical models with $\Omega_{\mathrm{m}}$ and $w$, we plug the equations (4) and (12), respectively, into the equation describing the luminosity distance for a flat FRWL metric, in units of megaparsecs

$d_{\mathrm{L}}(z ; \theta)=\frac{c(1+z)}{H_{0}} \int_{0}^{z} \frac{d z}{E(z ; \theta)}$

where the speed of light, $\mathrm{c}$, is in $\mathrm{km} \mathrm{s}^{-1}$ and the present Hubble parameter, $H_{0}$, in $\mathrm{km}(\mathrm{s} \mathrm{Mpc})^{-1}$. Here the vectors of parameters for each model are $\theta=\left(q_{0}, d q / d z\right)$ and $\theta=\left(\Omega_{\mathrm{m}}, w\right)$ respectively. For the gold sample data of Riess et al. (2004), we use the extinction-corrected distance moduli, $\mu^{\text {obs }}\left(z_{\mathrm{i}}\right)$ and associated errors, $\sigma_{\mathrm{i}}^{2}$. For the SNLS data of Astier et al.

1 Riess et al. (2004) presented 16 new Hubble Space Telescope (HST) SNIa, combined with 170 previously reported SNIa from ground-based data. They identified a widely used "highconfidence" subset, usually referred to as the gold sample, which includes 14 HST SNIa.

271 SNLS objects, plus 44 previously reported nearby objects.
(2005) we use the rest-frame-B-band magnitude at maximum light $m_{\mathrm{B}}^{*}\left(z_{\mathrm{i}}\right)$, the stretch factor $s_{\mathrm{i}}$ and the rest-frame color $c_{\mathrm{i}}$ to obtain $\mu^{\text {obs }}\left(z_{\mathrm{i}}\right)=m_{\mathrm{B}}^{*}\left(z_{\mathrm{i}}\right)-M+\alpha\left(s_{\mathrm{i}}-1\right)-\beta c_{\mathrm{i}}$. These values were derived from the light curves by Astier et al. (2005), who also provide best-fitting values for $\alpha=$ $1.52 \pm 0.14$ and $\beta=1.57 \pm 0.15$.

For both SNIa data sets, we have

$\chi^{2}\left(\theta ; m_{0}\right)=\sum_{\text {SNIa }} \frac{\left[\mu^{\text {th }}\left(z_{\mathrm{i}} ; \theta, \mu_{0}\right)-\mu^{\mathrm{obs}}\left(z_{\mathrm{i}} ; \theta, M\right)\right]^{2}}{\sigma_{\mathrm{i}}^{2}}$,

where the dispersion associated with each data point, $\sigma_{\mathrm{i}}^{2}=$ $\sigma_{\mu_{\mathrm{i}, \mathrm{obs}}}^{2}+\sigma_{\mathrm{int}, \mathrm{i}}^{2}+\sigma_{\mathrm{v}, \mathrm{i}}^{2}$. Here $\sigma_{\mu_{\mathrm{i}, \mathrm{obs}}}^{2}$ accounts for flux uncertainties, $\sigma_{\text {int,i }}^{2}$ accounts for intrinsic, systematic dispersion in SNIa absolute magnitudes and $\sigma_{\mathrm{v}, \mathrm{i}}^{2}$ accounts for systematic scatter due to peculiar velocities. The SNLS analysis includes an intrinsic dispersion of 0.13104 magnitudes $^{3}$ and a peculiar velocity scatter of $300 \mathrm{~km} / \mathrm{s}$. The gold sample analysis includes $400 \mathrm{~km} / \mathrm{s}$ peculiar velocity scatter, with an additional $2500 \mathrm{~km} / \mathrm{s}$ added in quadrature for high redshift SNIa.

We marginalise analytically over $m_{0}$

$\tilde{\chi}^{2}(\theta)=-2 \ln \int_{-\infty}^{\infty} \exp \left(-\frac{1}{2} \chi^{2}\left(\theta, m_{0}\right)\right) d m_{0}$

obtaining

$\tilde{\chi}^{2}=\ln \left(\frac{c}{2 \pi}\right)+a-\frac{b^{2}}{c}$,

where

$a=\sum_{\text {SNIa }} \frac{\left[5 \log _{10} D_{\mathrm{L}}\left(z_{\mathrm{i}} ; \theta\right)-m^{\mathrm{obs}}\left(z_{\mathrm{i}}\right)\right]^{2}}{\sigma_{\mathrm{i}}^{2}}$,

$b=\sum_{\text {SNIa }} \frac{5 \log _{10} D_{\mathrm{L}}\left(z_{\mathrm{i}} ; \theta\right)-m^{\mathrm{obs}}\left(z_{\mathrm{i}}\right)}{\sigma_{\mathrm{i}}^{2}}, \quad c=\sum_{\text {SNIa }} \frac{1}{\sigma_{\mathrm{i}}^{2}}$.

Note that the absolute value of $\chi^{2}=a-\left(b^{2} / c\right)$. For the analysis in the standard dynamic framework, our results agree with those of Riess et al. (2004) and Astier et al. (2005), and the comparison work of Nesseris \& Perivolaropoulos (2005).

\subsection{X-ray cluster data}

For the analysis of cluster X-ray gas mass fractions, we use the data of Allen et al. (2006), which contains 41 X-ray luminous, relaxed galaxy clusters, including 26 previously studied Allen et al. (2004). [Some of the original 26 have since been revisited by the Chandra X-ray observatory leading to improved constraints (for details see Allen et al. 2006).] The new X-ray data set spans a redshift interval $0.06<z<1.07$. Our analysis follows the method of Allen et al. (2004), fitting the apparent redshift evolution of the cluster gas fraction with the expression

$f_{\text {gas }}^{\text {ref }}\left(z_{\mathrm{i}}\right)=\mathcal{F} R^{\text {ref }}\left(z_{\mathrm{i}}\right), \quad R^{\text {ref }}\left(z_{\mathrm{i}}\right) \equiv\left[\frac{d_{\mathrm{A}}^{\mathrm{ref}}\left(z_{\mathrm{i}}\right)}{D_{\mathrm{A}}\left(z_{\mathrm{i}}\right)}\right]^{1.5}$,

where $\mathcal{F}=\left(b \Omega_{\mathrm{b}} H_{0}^{1.5}\right) /\left[(1+0.19 \sqrt{h}) \Omega_{\mathrm{m}}\right]$ is the normalization of the $f_{\text {gas }}(z)$ curve, $d_{\mathrm{A}}$ and $d_{\mathrm{A}}^{\text {ref }}(z)$ are the angular diameter distances $\left(d_{A}=d_{L} /(1+z)^{2}\right)$ to the clusters for

3 http://snls.in2p3.fr/conf/papers/cosmo1/ 
a given cosmology and for the reference $\Lambda$ CDM cosmology (with $H_{0}=70 \mathrm{~km}(\mathrm{~s} \mathrm{Mpc})^{-1}$ and $\Omega_{\mathrm{m}}=0.3$ ) respectively, and $D_{\mathrm{A}}=H_{0} d_{\mathrm{A}}$ is the $H_{0}$-free angular diameter distance. For the kinematical approach we treat the normalization $\mathcal{F}$ as a single 'nuisance' parameter, which we marginalize over in the MCMC chains.

For the dynamical analysis of the same X-ray data, we follow Allen et al. (2004) and employ Gaussian priors on the present value of the Hubble parameter $H_{0}=72 \pm 8 \mathrm{~km}(\mathrm{~s}$ $\mathrm{Mpc})^{-1}$ (Freedman et al. 2001), the mean baryon density $\Omega_{\mathrm{b}} h^{2}=0.0214 \pm 0.0020$ (Kirkman et al. 2003) and the X-ray bias factor $b=0.824 \pm 0.089$ [determined from the hydrodynamical simulations of Eke et al. (1998), including a 10 per cent allowance for systematic uncertainties]. The application of these priors leads to an additional constraint on $\Omega_{\mathrm{m}}$ from the normalization of the $f_{\text {gas }}(z)$ curve. Since the kinematical approach does not constrain $\Omega_{\mathrm{m}}$, the kinematical analysis does not involve these priors and draws information only from the shape of the $f_{\text {gas }}(z)$ curve. The dynamical analysis, in constrast, extracts information from both the shape and normalization.

\subsection{Markov Chain Monte Carlo analysis}

For both the kinematical and dynamical analyses, we sample the posterior probability distributions for all parameter spaces using a Markov Chain Monte Carlo (MCMC) technique. This provides a powerful tool for cosmological studies, allowing the exploration of large multi-dimensional parameter spaces. In detail, we use the Metropolis-Hastings algorithm implemented in the COSMOMC ${ }^{4}$ code of Lewis \& Bridle (2002) for the dynamic formalism, and a modified version of this code for the kinematic analysis.

Our analysis uses four MCMC chains for each combination of model and data. We ensure convergence by applying the Gelman-Rubin criterion (Gelman \& Rubin 1992), where the convergence is deemed acceptable if the ratio of the between-chain and mean-chain variances satisfies $R-1<0.1$. In general, our chains have $R-1 \ll 0.1$.

\subsection{Hypothesis testing in the kinematical analysis: how many model parameters are required?}

In the first case, we examined a kinematical model in which the deceleration parameter $q_{0}$ was included as the only interesting free parameter [see equation (4) with $q^{\prime}=0$ ]. This is hereafter referred to as the model $\mathcal{Q}$. As detailed in section 2, we next introduced the jerk parameter, $j$, as an additional free parameter, allowing it to take any constant value. We refer to this as model $\mathcal{J}$, which has the interesting free parameters, $q_{0}$ and $j$. Note that model $\mathcal{J}$ includes the set of possible $\Lambda$ CDM models, which all have constant $j=1$.

We next explored a series of models that allow for progressively more complicated deviations from $\Lambda$ CDM. In each case, the improvement obtained with the introduction of additional model parameters, has been gauged from the MCMC chains using a variety of statistical tests. In the first case, we follow a frequentist approach and use the F-test, for which

\footnotetext{
${ }^{4}$ http://cosmologist.info/cosmomc/
}

$F=\frac{\Delta \chi^{2}}{\chi_{\nu}^{2} \Delta m}$

where $\Delta \chi^{2}$ is the difference in the minimum $\chi^{2}$ between the two models, $\chi_{\nu}^{2}$ is the reduced $\chi^{2}\left(\chi^{2} / \nu\right.$, where $\nu$ is number of degrees of freedom of the fit, $d o f)$ of the final model, and $\Delta m$ is the difference in the number of free parameters in the two models. Given $\Delta m$ and $\nu$, we calculate the probability that the new model would give $\Delta \chi^{2} \geqslant F \chi_{\nu}^{2} \Delta m$ by random chance. This allows us to quantify the significance of the model extension.

The Bayesian Information Criterion (BIC) provides a more stringent model selection criterion and is an approximation to the Bayesian Evidence (Schwarz 1978). The BIC is defined as

$B I C=-2 \ln \mathcal{L}+k \ln N$

where $\mathcal{L}$ corresponds to the maximum likelihood obtained for a given model (thus, $-2 \ln \mathcal{L}$ is the minimum $\chi^{2}$ ), $k$ is the number of free parameters in the model and $N$ is the number of data points. Values for $\triangle B I C<2$ between two models are typically considered to represent weak evidence for an improvement in the fit. $\triangle B I C$ between 2 and 6 indicates 'positive evidence' for an improvement, and values greater than 6 signify 'strong evidence' for the model with the higher BIC (Jeffreys 1961; Kass \& Raftery 1995; Mukherjee et al. 1998; Liddle 2004).

Finally, we have compared the full posterior probability distributions for different models, using the Bayes Factor to quantify the significance of any improvement in the fit obtained. The Bayes Factor is defined as the ratio between the Bayesian evidences of the two models (Kass \& Raftery 1995). If $P(D \mid \theta, M)$ is the probability of the data $D$ given a model $M$, the Bayesian evidence is defined as the integral over the parameter space, $\theta$

$E(M) \equiv P(D \mid M)=\int d \theta P(D \mid \theta, M) P(\theta \mid M)$,

where $P(\theta \mid M)$ is the prior on the set parameters $\theta$, normalised to unity. We employ top hat priors for all parameters and evaluate the integrals using the MCMC samples:

$E(M) \sim \frac{1}{N \Delta \theta} \sum^{\mathrm{N}} P\left(D \mid \theta_{\mathrm{n}},\right)$

where $\Delta \theta$ is the volume in the parameter space selected to have probability 1 within the top hat priors, $N$ is the number of MCMC samples and $\theta_{\mathrm{n}}$ the sampled parameter space. Note that $\sum^{\mathrm{N}} P\left(D \mid \theta_{\mathrm{n}}\right)$ is the expected probability of the data in the posterior distribution (Lewis \& Bridle 2002). The evidence of the model $E(M)$ can be estimated trivially from the MCMC samples as the mean likelihood of the samples divided by the volume of the prior. It is clear, though, that this volume will depend on our selection of the top hat priors. In order to be as objective as possible, within the Bayesian framework, we use the same priors for parameters in common between the two models involved in a comparison. For parameters not in common, we calculate their volumes subtracting their maximum and minimum values in the MCMC samples.

The Bayes factor between two models $M_{0}$ and $M_{1}$ is $B_{01}=E\left(M_{0}\right) / E\left(M_{1}\right)$. If $\ln B_{01}$ is positive, $M_{0}$ is 'preferred' over $M_{1}$. If $\ln B_{01}$ is negative, $M_{1}$ is preferred over $M_{0}$. Following the scale of Jeffreys (1961), if $0<\ln B_{01}<1$ only 
Table 1. The marginalised median values and 68.3 per cent confidence intervals obtained analysing each data set and all three data sets together. We show these results for two kinematical models: using only $q_{0}(\mathcal{Q}$ model) and extending this parameter space with the jerk parameter $j\left(\mathcal{J}\right.$ model). We quote $\chi^{2}$ per degree of freedom for each model and three different statistical tests to quantify the significance of extending the parameter space from $\mathcal{Q}\left(q_{0}\right)$ to $\mathcal{J}\left(q_{0}, j\right)$. We quote the difference in $\Delta \chi_{\mathcal{J} \mathcal{Q}}^{2}$, the probability given by F-test, the difference in the Bayesian Information Criterion (BIC) and $\ln B_{\mathcal{J} \mathcal{Q}}$ (where $B_{\mathcal{J} \mathcal{Q}}$ is the Bayes factor between the two models). Note that combining all three data sets we obtain a significant preference for the $\mathcal{J}$ model within all three tests.

\begin{tabular}{|c|c|c|c|c|c|c|c|c|c|}
\hline Data set & \multicolumn{2}{|c|}{$\mathcal{Q}$ model } & \multicolumn{3}{|c|}{$\mathcal{J}$ model } & \multicolumn{4}{|c|}{ Improvement } \\
\hline Clusters & $-0.55 \pm 0.14$ & $39.6 / 39$ & $-0.61_{-0.41}^{+0.38}$ & $0.51_{-2.00}^{+2.55}$ & $39.6 / 38$ & 0.01 & $5.6 \%$ & -3.7 & -3.2 \\
\hline SNLS SNIa & $-0.417 \pm 0.062$ & $112.1 / 113$ & $-0.65 \pm 0.23$ & $1.32_{-1.21}^{+1.37}$ & $111.0 / 112$ & 1.1 & $69.4 \%$ & -3.6 & -2.5 \\
\hline Gold SNIa & $-0.289 \pm 0.062$ & $182.8 / 155$ & $-0.86 \pm 0.21$ & $2.75_{-1.10}^{+1.22}$ & $174.6 / 154$ & 8.2 & $99.1 \%$ & 3.1 & 1.2 \\
\hline
\end{tabular}

Table 2. The marginalised median values and 68.3 per cent confidence intervals obtained analysing all three data sets together. We show the results for the constant $j$ model (kinematical) and the constant $w$ model (dynamical) and their corresponding $\chi^{2}$ per degree of freedom.

\begin{tabular}{lcc}
\hline Approach & Model parameters & $\chi^{2} /$ dof \\
\hline Kinematical & $q_{0}=-0.81 \pm 0.14, j=2.16_{-0.75}^{+0.81}$ & $290.1 / 271$ \\
Dynamical & $\Omega_{\mathrm{m}}=0.306_{-0.040}^{+0.042}, w=-1.15_{-0.18}^{+0.14}$ & $291.7 / 272$ \\
\hline
\end{tabular}

a "bare mention" of the preference is considered warranted. If $1<\ln B_{01}<2.5$, the preference is regarded as of "substantial" significance. If $2.5<\ln B_{01}<5$ the significance is considered to be going from "strong" to "very strong".

\section{RESULTS}

\subsection{Comparison of constant jerk and constant $w$ models}

We first examine the statistical improvement obtained in moving from the simplest kinematical model $\mathcal{Q}=\left[q_{0}\right]$, in which $q_{0}$ is the only interesting free parameter, to model $\mathcal{J}=$ $\left[q_{0}, j\left(c_{0}\right)\right]$, where we include constant jerk $j=1+c_{0}$. The results obtained, using the three statistical tests described in subsection 4.4 applied to each data set alone and for all three data sets together are summarized in Table 1. None of the data sets, taken alone, strongly require the introduction of the jerk parameter, according to the Bayes factor test. (The 'gold' sample is the only data set that, on it's own, indicates a preference for model $\mathcal{J}$ over model $\mathcal{Q}$.) However, combining all three data sets, we obtain a strong preference for model $\mathcal{J}$ over model $\mathcal{Q}$, from all three statistical tests. Table 1 shows the mean marginalised parameters for each model and the $1 \sigma$ confidence levels. Combining all three data sets, we obtain tight constraints on $q_{0}=-0.81 \pm 0.14$ and $j=2.16_{-0.75}^{+0.81}$. This represents the first measurement of the jerk parameter from cosmological data. From our dynamical analysis of the same three data sets combined, we find $w=$ $-1.15_{-0.18}^{+0.14}$ and $\Omega_{\mathrm{m}}=0.306_{-0.040}^{+0.042}$ (see Table 2).
Figure 1 shows the constraints for the kinematical $\left(q_{0}, j\right.$; top left panel) and dynamical $\left(\Omega_{\mathrm{m}}, w\right.$; top right panel) models using all three data sets together. In both cases, the dashed lines indicates the expected range of results for $\Lambda$ CDM models (i.e. a cosmological constant). We find that both the kinematical and dynamical analyses of the combined data sets are consistent with the $\Lambda$ CDM model at about the $1 \sigma$ level.

It is important to recognise that the results from the kinematical and dynamical analyses constrain different sets of departures from $\Lambda$ CDM. We are using two simple, but very different parameterizations based on different underlying assumptions. The results presented in Figure 1 therefore provide interesting new support for the $\Lambda \mathrm{CDM}$ model.

The lower panels of figure 1 show the constraints obtained for the three data sets when analysed individually. It is important to note the consistent results from the independent SNIa and X-ray cluster data sets. Note that in the dynamical analysis, the X-ray data provide valuable additional constraints on $\Omega_{\mathrm{m}}$, when employing the $H_{0}$ and $\Omega_{\mathrm{b}} h^{2}$ priors. The overlap of all three data sets in both parameter spaces highlights the robustness of the measurements. Comparing the upper and lower panels of figure 1, we see how the combination of data sets significantly tightens the constraints.

\subsection{More complicated kinematical models}

For the combined data set, we have also searched for more complicated departures from $\Lambda$ CDM by including extra model parameters, as described in Section 3. We find no significant evidence for models more complicated than a constant jerk model. In particular, we find a negligible $\Delta \chi^{2}$ between models with constant jerk $\mathcal{J}=\left[q_{0}, j\left(c_{0}\right)\right]$ and the next most sophisticated model $\mathcal{J}_{1}=\left[q_{0}, j\left(a ; c_{0}, c_{1}\right)\right]$, and between the latter model and the next one, $\mathcal{J}_{2}=\left[q_{0}, j\left(a ; c_{0}, c_{1}, c_{2}\right)\right]$.

It is, however, interesting to plot the differences between the constraints obtained for each model. Figure 2 shows the current $1 \sigma$ and $2 \sigma$ constraints around the median values of $j(a)$ at different scale factors, $a$, over the range where we have data $[0.36,1]$. The green, lighter contours show the constraints for the $\mathcal{J}_{1}$ model and the red, darker contours for the $\mathcal{J}$ model. From this figure it is clear that current data 

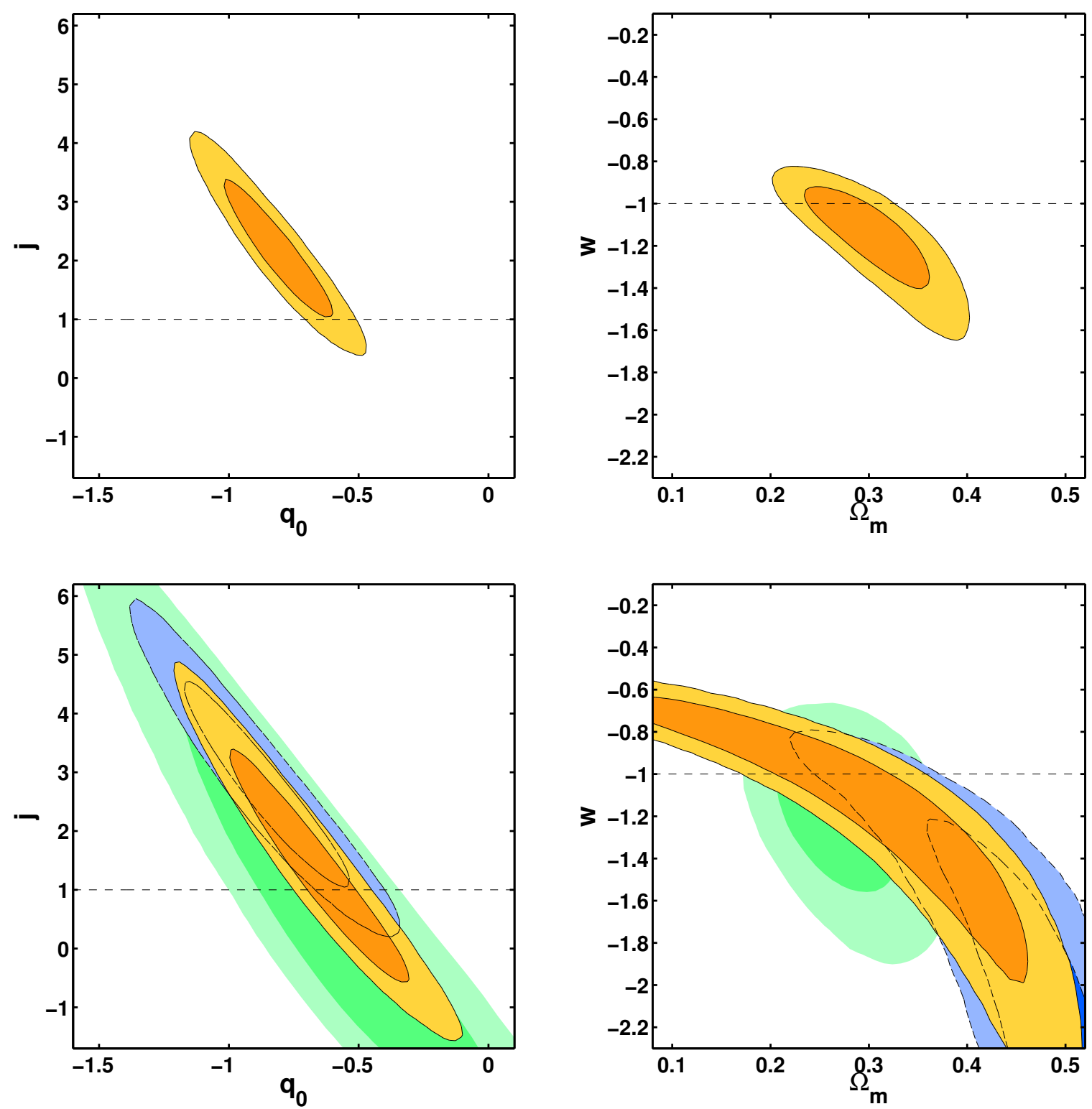

Figure 1. A summary of the results from the kinematical (left panels) and dynamical (right panels) analyses. The top left panel shows the 68.3 and 95.4 per cent confidence limits in the $\left(q_{0}, j\right)$ plane for the kinematical model with a constant jerk, $j$, obtained using all three data sets: both SNIa data sets (Riess et al. 2004; Astier et al. 2005) and the cluster $f_{\text {gas }}$ data of Allen et al. (2006). The top right panel shows the results in the $\left(\Omega_{\mathrm{m}}, w\right)$ plane obtained using the same three data sets and assuming HST, BBNS and $b$ priors. (Note that the kinematical analysis does not use the HST, BBNS and $b$ priors). The dashed lines show the expectation for a cosmological constant model in both formalisms $(j=1, w=-1$, respectively). The bottom panels show the confidence contours in the same planes for the individual data sets: the SNLS SNIa data (orange contours), the Riess et al. (2004) 'gold' SNIa sample (blue contours) and the cluster $f_{\text {gas }}$ data (green contours). Here, the dashed lines again indicate the cosmological constant model.

provide the best constraints around $a \sim 0.77$, i.e. $z \sim 0.3$, and that at higher and lower redshift more data are required. For the low redshift range, the forthcoming SDSS II SNIa data will be helpful. For the high redshift range, new HST SNIa and further X-ray cluster data should be available in the near future. In the longer term, SNIa data from the Large Synaptic Survey Telescope (LSST) ${ }^{5}$ and the Supernovae Acceleration Probe (SNAP) ${ }^{6}$, and X-ray cluster data from Constellation- $\mathrm{X}^{7}$ should provide tight constraints on both $j(a)$ and $w(a)$. Future galaxy redshift surveys covering a high redshift range will also help to tighten these constraints, using the baryon oscillation experiment (Eisenstein et al. 2005; Cole et al. 2005).

\footnotetext{
5 http://www.lsst.org/lsst_home.shtml

6 http://snap.lbl.gov/
} 


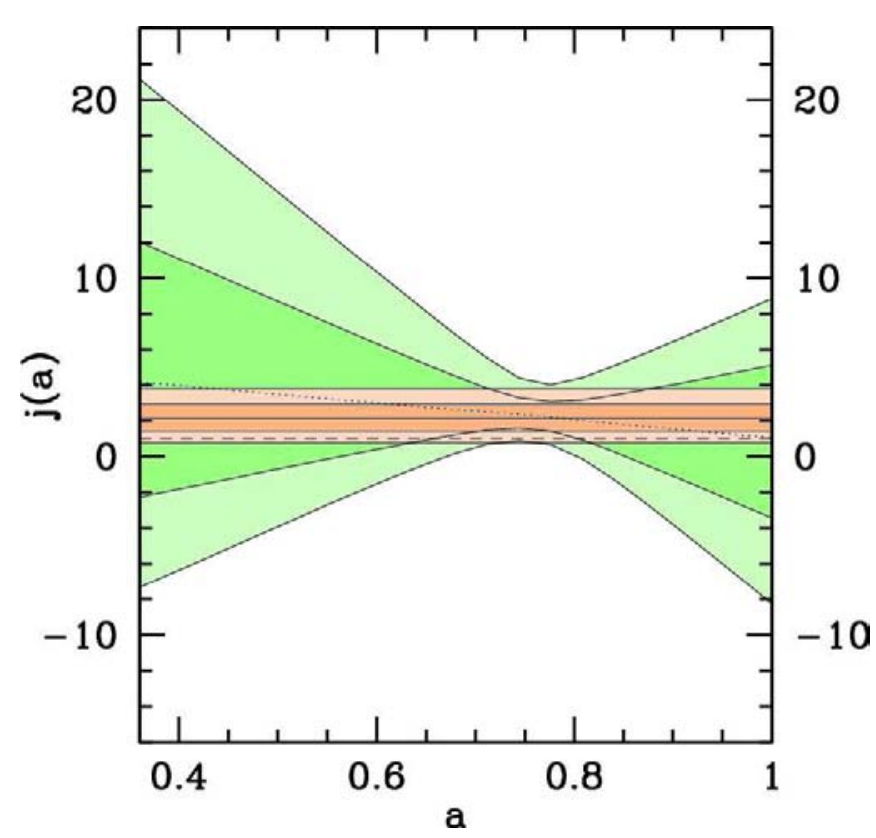

Figure 2. The 68.3 and 95.4 per cent confidence variations about the median values for $j(a)$ as a function of the scale factor $a$, over the range where we have data $[0.36,1]$. Results are shown for the constant jerk model (model $\mathcal{J}$ ) (red, darker contours) and $\mathcal{J}_{1}$ model (green, lighter contours). In both cases, the constraints for all three data sets have been combined. The dashed line indicates the expectation, $j=1$ (constant) for a cosmological constant $(\Lambda \mathrm{CDM})$ model.

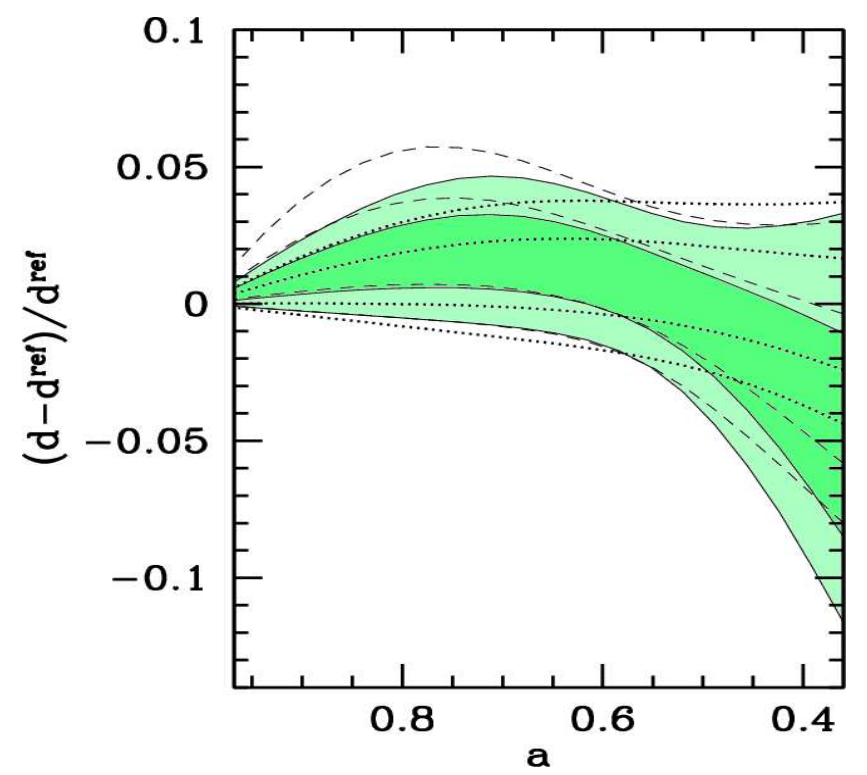

Figure 3. The 68.3 and 95.4 per cent confidence limits on the offset in distance as a function of scale factor, relative to the reference $\Lambda \mathrm{CDM}$ cosmology, for both the kinematical (constant $j$; green, shaded curves) and dynamical (constant $w$; dotted and dashed curves) analyses. The dotted curves show the results for the dynamical analysis in which the additional constraint on $\Omega_{\mathrm{m}}$ from the normalization of the $f_{\text {gas }}$ curve is used. The dashed curve is for a dynamical analysis where this extra constraint on the normalization is ignored. The same MCMC samples used to construct Fig 1 have been used.

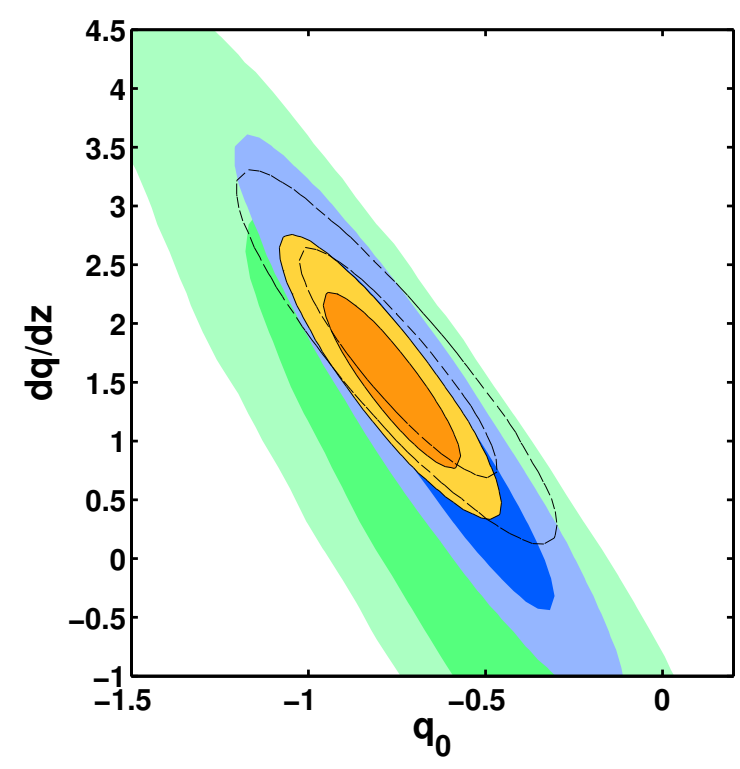

Figure 4. The 68.3 and 95.4 per cent confidence limits in the $\left(q_{0}, d q / d z\right)$ plane obtained using the SNIa data from the first year of the SNLS (Astier et al. 2005) (blue contours), the 'gold' sample of Riess et al. (2004) (dashed contours), the cluster $f_{\text {gas }}$ data of Allen et al. (2006) (green contours) and the combination of all three data sets (orange contours).

\subsection{Comparison of distance measurements}

It is interesting to compare directly the distance curves for the kinematical (constant $j$ ) and dynamical (constant $w$ ) models, as determined from the MCMC chains. Fig 3 shows the 68.3 and 95.4 per cent confidence limits on the offset in distance, as a function of scale factor, relative to a reference $\Lambda$ CDM cosmology with $\Omega_{\mathrm{m}}=0.27, \Omega_{\Lambda}=0.73$. We see that the kinematical and dynamical results occupy very similar, though not identical, loci in the distance-scale factor plane. For the dynamical analysis, the addition of the extra constraint on $\Omega_{\mathrm{m}}$ from the normalization of the $f_{\text {gas }}$ curve tightens the constraints and pushes the results in a direction slightly more consistent with the reference $\Lambda \mathrm{CDM}$ cosmology.

\subsection{Comparison with Riess et al. (2004)}

Finally, for comparison purposes, we also present the results obtained using the linear parameterization of $q(z)$ described by equation (3) and used by Riess et al. (2004). Figure 4 shows the constraints in the plane $\left(q_{0}, d q / d z\right)$ determined from each data set, and by combining the three data sets (solid, orange contours). It is clear that the constraints from the three independent data sets overlap and that by combining them we obtain significantly tighter results than using the 'gold' sample alone.

7 http://constellation.gsfc.nasa.gov/ 


\section{THE DISTANCE TO THE LAST SCATTERING SURFACE}

Finally, we note that there is one further pseudo-distance measurement available to us - the distance to the last scattering surface from CMB data. Although this is not a purely kinematical data point, for illustration purposes we show the constraints on $j(a)$ that can be achieved if one is willing to make extra assumptions and include this measurement. The extra assumptions involved, though strong, are well-motivated. In detail, in order to use the distance to last scattering, we assume that dark matter behaves like standard cold dark matter at all redshifts, an assumption well tested by e.g. galaxy cluster, weak lensing and galaxy redshift surveys at low redshifts and $\mathrm{CMB}$ experiments at high- $z$. We also assume that pre-recombination physics can be well described by a standard combination of cold dark matter, a photon-baryon fluid and neutrinos, and that any early dark energy component has a negligible affect on the dynamics. With these assumptions, one can construct the comoving angular diameter distance to the last scattering surface from $d_{\mathrm{A}}=r_{\mathrm{s}}\left(a_{d e c}\right) / \theta_{\mathrm{A}}$, where $r_{\mathrm{s}}\left(a_{r e c}\right)$ and $\theta_{\mathrm{A}}$ are the comoving sound horizon at decoupling and the characteristic angular scale of the acoustic peaks, respectively. For a geometrically flat Universe with a negligible early dark energy component, we calculate the sound horizon at decoupling as (Verde et al. 2003)

$r_{\mathrm{s}}\left(a_{\mathrm{dec}}\right) \simeq \int_{0}^{a_{\mathrm{dec}}} \frac{c_{\mathrm{s}}(a)}{H_{0}\left(\Omega_{\mathrm{m}} a+\Omega_{\mathrm{rad}}\right)^{1 / 2}} d a$,

where $c_{\mathrm{s}}(a)=c /\left[1+\left(3 \Omega_{\mathrm{b}} a\right) /\left(4 \Omega_{\gamma}\right)\right]$ is the sound speed in the photon-baryon fluid, $\Omega_{\mathrm{rad}}=\Omega_{\gamma}+\Omega_{\nu}$ is the present radiation energy density, and $\Omega_{\gamma}$ and $\Omega_{\nu}$ are the present photon and neutrino energy densities, respectively. We use our $\mathrm{X}$-ray galaxy cluster data, assuming HST, BBNS and $b$ priors, to determine $\Omega_{m}=0.27 \pm 0.04$ (Allen et al. 2006; note that this constraint mainly comes from low-redshift clusters). We also use the COBE measurement of the CMB temperature $T_{0}=2.725 \pm 0.002 \mathrm{~K}$ (Mather et al. 1999) and a standard three neutrino species model with negligible masses to obtain $\Omega_{\text {rad }}$. For these constraints, we obtain $r_{\mathrm{s}}\left(z_{\mathrm{dec}}\right) \simeq 146 \pm 10 \mathrm{Mpc}$.

From Hinshaw et al. (2006) we have the multipole of the first acoustic peak $l_{1}=220.7 \pm 0.7$. This is related to $l_{\mathrm{A}}$ by a shift $\phi, l_{1}=l_{\mathrm{A}}(1-\phi)$. Using the fitting formula of Doran \& Lilley (2002), the BBNS prior for $\Omega_{\mathrm{b}} h^{2}$, a scalar spectral index $n_{\mathrm{s}}=0.95 \pm 0.02$ (Spergel et al. 2006) and assuming no early dark energy, we find $\theta_{\mathrm{A}}=0.6 \pm 0.01$ degrees. We then obtain a pseudo-model-independent distance to decoupling, $d\left(z_{\mathrm{dec}}\right) \simeq 13.8 \pm 1.1 \mathrm{Gpc}$, where $z_{\mathrm{dec}}=1088$ (Spergel et al. 2006).

Fig 5 shows the tightening of the constraints obtained using this "data-point-prior" at high redshift ${ }^{8}$. Note that

\footnotetext{
8 Note that extending the analysis to the decoupling redshift $z_{\text {dec }}=1088$ means that the radiation density becomes nonnegligible. Although, obviously $j$ can still be calculated as usual, $j^{\Lambda C D M}$ will not equal 1 at these redshifts. However, the $\Lambda \mathrm{CDM}$ model can then be almost perfectly described as $j^{\Lambda C D M}(a)=$ $1+2 /\left(1+\left(a / a_{\text {eq }}\right)\right)$ (see Amin et al. 2006, for details) where $a_{\text {eq }}$ is the mean marginalised scale factor at equality, from WMAP data. We have explicitly verified that, within the $1 \sigma$ values of $a_{\text {eq }}$, sys-
}

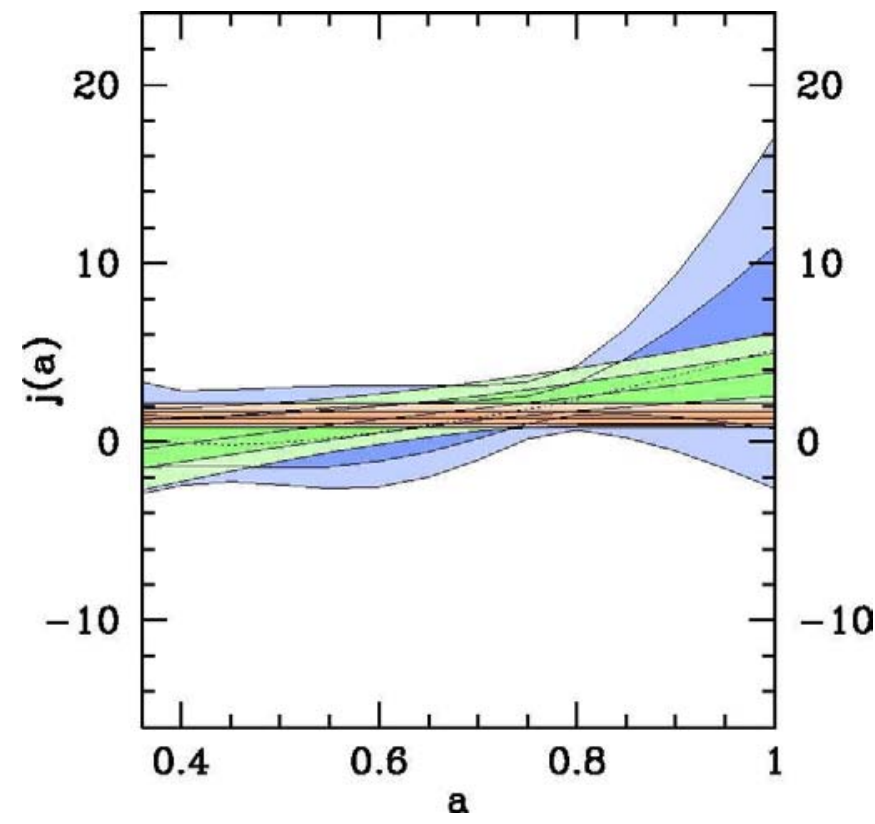

Figure 5. $1 \sigma$ and $2 \sigma$ constraints around the median values of $j(a)$ along the scale factor $a$ in the range where we have data $[0.36,1]$. This figure shows the same models than figure 2 plus the $\mathcal{J}_{2}$ model and uses the CMB prior described at the text. Here the dotted line is for the $j(a)$ median values for the $\mathcal{J}_{1}$ model. Note that this figure and figure 2 have the same scale for comparison purposes.

figure 5 is on the same scale as figure 2 and shows the same models: $\mathcal{J}$ (red, darker contours) and $\mathcal{J}_{1}$ (green, lighter contours) plus the $\mathcal{J}_{2}$ model (blue contours). The prior information at high redshift, from the distance to last scattering, tightens the constraints significantly. Evidently, the constraints from the kinematic analysis are sensitive to the data quality at high redshift.

\section{CONCLUSIONS}

We have developed a new kinematical approach to study the expansion of the history of the Universe, building on the earlier work of Blandford et al. (2004). Our technique uses the parameter space defined by the current value of the cosmic deceleration parameter $q_{0}$ and the jerk parameter $j$, where $q$ and $j$ are the dimensionless second and third derivatives of the scale factor with respect to cosmic time. The use of this $\left(q_{0}, j\right)$ parameter space provides a natural framework for kinematical studies. In particular, it provides a simple prescription for searching for departures from $\Lambda$ CDM, since the complete set of $\Lambda \mathrm{CDM}$ models are characterized by $j=1$ (constant).

We have applied our technique to the three best available sets of redshift-independent distance measurements, from type Ia supernovae studies (Riess et al. 2004; Astier et al. 2005) and measurements of the X-ray gas mass fraction in X-ray luminous, dynamically relaxed galaxy clusters (Allen et al. 2006). Assuming geometric flatness, we measure

tematic offsets due to the affects of radiation have a negligible effect on the derived distances. 
$q_{0}=-0.82 \pm 0.14$ and $j=2.16_{-0.75}^{+0.81}$ (Figure 1 ). Note that this represents the first measurement of the cosmic jerk parameter, $j$. A more standard, dynamical analysis of the same data gives $w=-1.15_{-0.18}^{+0.14}$ and $\Omega_{\mathrm{m}}=0.306_{-0.040}^{+0.042}$, also assuming flatness and HST, BBNS and $b$ priors (Figure 1). Both sets of results are consistent with the standard $\Lambda \mathrm{CDM}$ paradigm, at about the $1 \sigma$ level.

In comparison to standard, dynamical approaches, our kinematical framework provides a different set of simple models and involves fewer assumptions. In particular, kinematical analyses such as that presented here do not assume a particular gravity theory. The combination of the kinematical and dynamical approaches therefore provides important, complementary information for investigating late time cosmic acceleration. The fact that both the kinematical and dynamical results presented here are consistent with $\Lambda \mathrm{CDM}$ provides important additional support for that model. The fact that the two independent sets of distance measurements, from X-ray galaxy clusters and supernovae, are individually consistent with $\Lambda \mathrm{CDM}$, is reassuring (Figure 1).

We have searched for departures from $\Lambda$ CDM using a new scheme based on the introduction of Chebyshev polynomials. These orthonormal functions allow us to expand any deviation from $\Lambda \mathrm{CDM}, \Delta j(a ; \mathcal{C})$, as a linear combination of polynomials. We use the coefficients of these polynomials, $\mathcal{C}$, as fit parameters. The current data provide no evidence for a dependence of $j$ on $a$ more complicated than a constant value. However, higher order terms may be required to describe future data sets. In that case, our scheme has the advantage that, over a finite interval and using enough high order terms, it will provide an acceptable global approximation to the true underlying shape. Note that this scheme is also applicable to dynamical studies of the evolution of the dark energy equation of state, $w(a)$.

We suggest that future studies should endeavour to use both kinematical and dynamical approaches where possible, in order to extract the most information from the data. The two approaches have different strengths, can be applied to with a variety of data sets, and are highly complementary. The combination of techniques may be especially helpful in to distinguish between an origin for cosmic acceleration that lies with dark energy (i.e. a new energy component to the Universe) from modifications to General Relativity.

\section{ACKNOWLEDGMENT}

We acknowledge helpful discussions with A. Frolov and technical support from G. Morris. The computational analysis was carried out using the KIPAC XOC compute cluster at SLAC. SWA acknowledges support from the National Aeronautics and Space Administration through Chandra Award Number DD5-6031X issued by the Chandra X-ray Observatory Center, which is operated by the Smithsonian Astrophysical Observatory for and on behalf of the National Aeronautics and Space Administration under contract NAS803060. RDB acknowledges support from National Science Foundation grant AST05-07732. This work was supported in part by the U.S. Department of Energy under contract number DE-AC02-76SF00515.

\section{REFERENCES}

Alam U., Sahni V., Saini T. D., Starobinsky A. A., 2003, MNRAS, 344, 1057

Allen S. W., et al., 2006, in preparation

Allen S. W., Schmidt R. W., Ebeling H., Fabian A. C., van

Speybroeck L., 2004, MNRAS, 353, 457

Allen S. W., Schmidt R. W., Fabian A. C., 2002, MNRAS, 334, L11

Allen S. W., Schmidt R. W., Fabian A. C., Ebeling H., 2003, MNRAS, 342, 287

Amin M. A., Blandford R. D., Wang P., 2006, in preparation

Armendariz-Picon C., Mukhanov V., Steinhardt P. J., 2000, Phys. Rev. Lett., 85, 4438

Armendariz-Picon C., Mukhanov V., Steinhardt P. J., 2001, Phys. Rev., D63, 103510

Astier P., et al., 2005, astro-ph/0510447

Bagla J. S., Jassal H. K., Padmanabhan T., 2003, Phys. Rev., D67, 063504

Barreiro T., Copeland E. J., Nunes N. J., 2000, Phys. Rev., D61, 127301

Bento M. C., Bertolami O., Sen A. A., 2002, Phys. Rev., D66, 043507

Blandford R. D., Amin M., Baltz E. A., Mandel K., Marshall P. J., 2004, in "NOAO Workshop on Observing Dark Energy" "cosmokinetics"

Borgani S., Rosati P., Tozzi P., Stanford S. A., Eisenhardt P. R., Lidman C., Holden B., Della Ceca R., Norman C., Squires G., 2001, ApJ, 561, 13

Cabre A., Gaztanaga E., Manera M., Fosalba P., Castander F., 2006, astro-ph/0603690

Caldwell R., Dave R., Steinhardt P., 1998, Phys. Rev. Lett., 80,1582

Caldwell R. R., Doran M., 2005, Phys. Rev., D72, 043527

Capozziello S., Carloni S., Troisi A., 2003, astro$\mathrm{ph} / 0303041$

Carroll S. M., Duvvuri V., Trodden M., Turner M. S., 2004, Phys. Rev., D70, 043528

Carroll S. M., et al., 2005, Phys. Rev., D71, 063513

Carroll S. M., Hoffman M., Trodden M., 2003, Phys. Rev., D68, 023509

Chiba T., Okabe T., Yamaguchi M., 2000, Phys. Rev., D62, 023511

Cole S., et al., 2005, MNRAS, 362, 505

Copeland E. J., Garousi M. R., Sami M., Tsujikawa S., 2005, Phys. Rev., D71, 043003

Copeland E. J., Liddle A. R., Wands D., 1998, Phys. Rev., D57, 4686

Copeland E. J., Sami M., Tsujikawa S., 2006, hepth/0603057

Corasaniti P. S., Kunz M., Parkinson D., Copeland E. J., Bassett B. A., 2004, Phys. Rev., D70, 083006

Croft R. A. C., et al., 2002, ApJ, 581, 20

Deffayet C., Dvali G., Gabadadze G., 2002, Phys. Rev.D, 65, 044023

Deffayet C., Landau S. J., Raux J., Zaldarriaga M., Astier P., 2002, Phys. Rev., D66, 024019

Doran M., Lilley M., 2002, MNRAS, 330, 965

Dvali G. R., Gabadadze G., Porrati M., 2000, Phys. Lett., B485, 208

Eisenstein D. J., et al., 2005, ApJ, 633, 560 
Eke V. R., Navarro J. F., Frenk C. S., 1998, ApJ, 503, 569 Elgaroy O., Multamaki T., 2006, astro-ph/0603053

Ettori S., Tozzi P., Rosati P., 2003, Astron. Astrophys., 398, 879

Fosalba P., Gaztanaga E., Castander F., 2003, ApJ, 597, L89

Freedman W., et al., 2001, ApJ, 553, 47

Gelman A., Rubin D. B., 1992, Statis. Sci., 7, 457

Gong Y., Wang A., 2006, astro-ph/0601453

Guo Z.-K., Zhu Z.-H., Alcaniz J. S., Zhang Y.-Z., 2006, astro-ph/0603632

Hinshaw G., et al., 2006, astro-ph/0603451

Hoekstra H., Yee H. K. C., Gladders M. D., 2002, ApJ, 577,595

Hu W., 2005, Phys. Rev., D71, 047301

Jarvis M., Jain B., Bernstein G., Dolney D., 2005, astro$\mathrm{ph} / 0502243$

Jassal H. K., Bagla J. S., Padmanabhan T., 2005, MNRAS, 356, L11

Jeffreys H., 1961, Theory of probability. Oxford Univ. Press, Oxford

Kamenshchik A. Y., Moschella U., Pasquier V., 2001, Phys. Lett., B511, 265

Kass R. E., Raftery A. E., 1995, J. Am. Stat. Assn.

Kirkman D., Tytler D., Suzuki N., O'Meara J. M., Lubin D., 2003, ApJ S., 149, 1

Knop R. A., et al., 2003, ApJ, 598, 102

Lewis A., Bridle S., 2002, Phys. Rev., D66, 103511

Liddle A. R., 2004, MNRAS, 351, L49

Linder E. V., 2006, Phys. Rev., D73, 063010

Maartens R., Majerotto E., 2006, astro-ph/0603353

Mather J. C., Fixsen D. J., Shafer R. A., Mosier C., Wilkinson D. T., 1999, ApJ, 512, 511

Mena O., Santiago J., Weller J., 2006, Phys. Rev. Lett., 96, 041103

Mukherjee S., Feigelson E. D., Jogesh Babu G., Murtagh F., Fraley C., Raftery A., 1998, ApJ, 508, 314

Navarro I., Van Acoleyen K., 2005, gr-qc/0512109

Nesseris S., Perivolaropoulos L., 2005, Phys. Rev., D72, 123519

Perlmutter S., et al., 1999, ApJ, 517, 565

Rapetti D., Allen S. W., Weller J., 2005, MNRAS, 360, 555

Reiprich T. H., Böhringer H., 2002, ApJ, 567, 716

Riess A. G., et al., 1998, ApJ, 116, 1009

Riess A. G., et al., 2004, ApJ, 607, 665

Sahni V., Saini T. D., Starobinsky A. A., Alam U., 2003, JETP Lett., 77, 201

Schuecker P., Caldwell R. R., Bohringer H., Collins C. A., Guzzo L., 2003, Astron. Astrophys., 402, 53

Schwarz G., 1978, Ann. Stat.

Scranton R., et al., 2003, astro-ph/0307335

Seljak U., et al., 2005, Phys. Rev., D71, 103515

Shapiro C., Turner M. S., 2005, astro-ph/0512586

Spergel D. N., et al., 2003, ApJ S., 148, 175

Spergel D. N., et al., 2006, astro-ph/0603449

Steinhardt P. J., Wang L.-M., Zlatev I., 1999, Phys. Rev., D59, 123504

Tegmark M., et al., 2004, Phys. Rev., D69, 103501

Upadhye A., Ishak M., Steinhardt P. J., 2005, Phys. Rev., D72, 063501

van Waerbeke L., et al., 2000, Astron. Astrophys., 358, 30
Van Waerbeke L., Mellier Y., Hoekstra H., 2005, Astron. Astrophys., 429, 75

Verde L., et al., 2003, ApJ S., 148, 195

Viel M., Weller J., Haehnelt M., 2004, MNRAS, 355, L23

Vikman A., 2005, Phys. Rev., D71, 023515

Visser M., 2004, Class. Quant. Grav., 21, 2603

Voevodkin A., Vikhlinin A., 2004, ApJ, 601, 610

Vollick D. N., 2003, Phys. Rev., D68, 063510

Weller J., Lewis A. M., 2003, MNRAS, 346, 987

Zhao G.-B., Xia J.-Q., Feng B., Zhang X., 2006, astro$\mathrm{ph} / 0603621$

Zhao G.-B., Xia J.-Q., Li M., Feng B., Zhang X., 2005, Phys. Rev., D72, 123515

Zlatev I., Wang L.-M., Steinhardt P. J., 1999, Phys. Rev. Lett., 82, 896 